\title{
Study of Ethanol Vapour Sensing Behaviour by Polypyrrole-multiwall Carbon Nanotubes Nanocomposites
}

\author{
Sanjay G. Bachhav and Dilip R. Patil* \\ Nanomaterial Research Laboratory, R. C. Patel Arts, Commerce and Science College, \\ Shirpur 425405, Maharashtra, India \\ ${ }^{*}$ Corresponding author: dr.drpatil@gmail.com
}

Published online: 25 August 2018

To cite this article: Bachhav, S. G. \& Patil, D. R. (2018). Study of ethanol vapour sensing behaviour by polypyrrole-multiwall carbon nanotubes nanocomposites. J. Phys. Sci., 29(2), 137-152, https://doi.org/10.21315/jps2018.29.2.9

To link to this article: https://doi.org/10.21315/jps2018.29.2.9

\begin{abstract}
Polypyrrole-multiwall carbon nanotubes (PPy/MWCNT) nanocomposites were synthesised by in-situ chemical oxidative polymerisation method. The MWCNTs were functionalised prior to the formation of nanocomposites. These nanocomposites were characterised by field emission scanning electron microscopy (FESEM), X-ray diffraction (XRD), Fourier transform infrared (FTIR) spectroscopy and thermogravimetric analysis (TGA) to study the effect of incorporation of functionalised MWCNT in PPy matrix. The results showed the successful formation of PPy/MWCNT nanocomposite and there is significant interaction between PPy and MWCNTs. The response of the prepared PPy/ MWCNT nanocomposites sensors was studied in the form of sensitivity towards ethanol vapours. Results showed that the response increases with ethanol concentration and it is also affected by the MWCNT content in PPy matrix.
\end{abstract}

Keywords: Conducting polymers, polypyrrole, carbon nanotubes, in-situ polymerisation, gas sensing

\section{INTRODUCTION}

Carbon nanotubes (CNTs), discovered by Iijima in 1991, have promising applications in the field of nanoscience and nanotechnology due to their excellent electrical, thermal and extraordinary mechanical properties, and their suitability as conducting filler in polymer composite. ${ }^{1}$ Owing to their large surface-to-volume ratio and presence of defects and porous structure, CNTs offer effective binding sites for gas molecules. The gas molecules adsorbed on the CNT surface influence its electrical parameters such as resistance and capacitance. CNTs demonstrate great potential in next-generation sensor technology because of their stable properties. ${ }^{2}$

(C) Penerbit Universiti Sains Malaysia, 2018. This work is licensed under the terms of the Creative Commons Attribution (CC BY) (http://creativecommons.org/licenses/by/4.0/). 
Conducting polymers have received much attention due to their potential applications in electronic devices, super capacitor, batteries, sensors and many other fields. ${ }^{3}$ Among several conducting polymers, polypyrrole (PPy) has been studied extensively in the recent years due to its high electrical conductivity along with high stability upon exposure to ambient conditions, easy synthesis, good environmental stability and cost effectiveness. ${ }^{4-6}$ Also, PPy possesses several excellent qualities and stimulus-responsive properties that make it a very promising "smart" nanomaterial. ${ }^{7}$

Over the last two decades, nanocomposites based on CNTs and conducting polymers have been widely investigated for developing new class of multifunctional advanced materials. Such materials possess unique and superior properties due to the synergistic effects resulting from combination of these two kinds of material. ${ }^{8}$ The PPy-CNT nanocomposites are synthesised especially with core-shell morphology by various methods. ${ }^{9-13}$ Among these methods, chemical oxidisation is simple, fast, cheap and easily scaled up. ${ }^{14}$

The importance of ethanol detection is well understood as ethanol is common volatile organic compound (VOC) in the industry for alimental manufacturing, cosmetic production and organic synthesis. The ethanol sensors find applications in various areas such as alcohol detection in driving enforcement, monitoring of fermentation and other processes in chemical industries. The exhaled breath of a human being contains many VOCs such as acetone, methanol, ethanol, propanol, etc., and thus analysis of such VOC present in breath has proven to be important for reliable clinical diagnosis of lung cancer. ${ }^{15-17}$ Also, breath analysis provides a fast and non-invasive diagnostic technique, as it can be done directly in the gas state. Therefore, ethanol is one of the biomarkers in lung cancer detection.

The use of conductive polymer-carbon nanotube nanocomposites has gained a lot of attention due to their outstanding properties such as having very high specific surface area, small size, high electrical conductivity and thermal stability. An immediate response of their electrical properties with the adsorption of gas molecules makes CNT a very important sensing material. ${ }^{18,19}$ Several types of materials are used to fabricate the VOC sensors composing "e-noses." ${ }^{20-22}$ However, the use of conductive polymer-based nanocomposite material has the advantage of operating at a room temperature and their selectivity can be tailored easily by changing the chemical nature of their polymer matrix.

In the present work, synthesis and characterisation of PPy/MWCNT nanocomposite was carried out by varying the MWCNT content, keeping PPy composition constant. MWCNTs were used as nanofillers in polymer matrix and serve as conducting channels. Their chemo-electrical behaviour of nanocomposite to ethanol vapour 
over a wide range was investigated at room temperature and the effect of MWCNT filler content on its sensing behaviour was also studied.

\section{EXPERIMENTAL}

\subsection{Synthesis of PPy/MWCNT Nanocomposites}

MWCNTs used in this work were acquired from Sigma Aldrich. The pyrrole monomer used was received from Spectrochem (Mumbai, India), while ammonium persulfate (APS) used as oxidising agents was acquired from SDFC (Mumbai, India). All these reagents used were analytical grade.

MWCNTs were functionalised by using acid boiling reflux treatment. In this method, $500 \mathrm{mg}$ MWCNTs were suspended in a mixture of $\mathrm{H}_{2} \mathrm{SO}_{4} / \mathrm{HNO}_{3}(3: 1)$. The sample was stirred magnetically at $80^{\circ} \mathrm{C}$ for $12 \mathrm{~h}$. The final functionalised MWCNT product was obtained by filtering, washing and drying the mixture.

Pristine PPy and PPy/MWCNT nanocomposite samples were prepared by in-situ chemical oxidative polymerisation. In the synthesis of PPy/MWCNT nanocomposite, a certain amount of functionalised MWCNTs and $50 \mu 1$ Triton $\mathrm{X}-100$ were dispersed into $50 \mathrm{ml}$ distilled water by probe sonicating for $1 \mathrm{~h}$. The Triton X-100 is surfactant and used to enhance the dispersion of MWCNTs into water. The pyrrole monomer $(2 \mathrm{ml})$ was added and the mixture was magnetically stirred for $10 \mathrm{~min}$. A $1 \mathrm{M} \mathrm{HCl}(50 \mathrm{ml})$ was added to the solution. An aqueous solution of $0.55 \mathrm{M}$ APS $(50 \mathrm{ml})$ solution was prepared and added drop-by-drop into pyrrole-MWCNT with constant stirring for $30 \mathrm{~min}$. The stirring was continued for next $12 \mathrm{~h}$ and let the dispersion stand for $4 \mathrm{~h}$ in ice-water bathing. Finally, the PPy/MWCNT nanocomposite powder was obtained by filtering the mixture, washing with water, ethanol and methanol, and drying in an oven at $60^{\circ} \mathrm{C}$ for $12 \mathrm{~h}$.

By varying the MWCNT contents (such as $5 \mathrm{mg}, 10 \mathrm{mg}, 25 \mathrm{mg}$ and $50 \mathrm{mg}$ ), four $\mathrm{PPy} / \mathrm{MWCNT}$ composite samples were prepared, keeping molar concentration of PPy fixed. The resulting composite samples were named as PPy/MWCNT5, PPy/ MWCNT10, PPy/MWCNT25 and PPy/MWCNT50. The pristine PPy sample was also prepared by the similar procedure as described above except use of MWCNTs and Triton X-100.

\subsection{Characterisations}

The morphology of PPy and its nanocomposite samples was analysed by Hitachi S4800 field emission scanning electron microscopy (FESEM). X-ray diffraction 
(XRD) pattern of all the samples was recorded using Bruker D8 Advance diffractometer with $\mathrm{Cu} \mathrm{K} \alpha$ radiation. Fourier transform infrared (FTIR) spectrum of samples was obtained using a Shimadzu IR spectrometer in $\mathrm{KBr}$ medium to ensure functional groups. TGA was performed using a Perkin Elmer 4000 thermogravimetric analyser in temperature range from room temperature to $700^{\circ} \mathrm{C}$ with heating rate of $20^{\circ} \mathrm{C} \mathrm{min}^{-1}$ to study the thermal degradation of the samples.

\subsection{Sensor Preparation}

The sensor was made by depositing nanocomposite material on interdigited electrode (IDE) made from copper clad. The interdigited electrode was made from glass epoxy copper clad of dimension $2 \mathrm{~cm} \times 1.3 \mathrm{~cm}$. The track size as well as spacing between tracks of IDE electrode is $1 \mathrm{~mm}$. The nanocomposite powder was dissolved in N,N dimethylformide (DMF) and drop casted on interdigited electrode plate made form glass epoxy copper clad. The sensor was then kept in oven at $60^{\circ} \mathrm{C}$ temperature for $4 \mathrm{~h}$ so as to evaporate and to completely remove the remnant DMF. The IDE sensor is schematically represented in Figure 1.

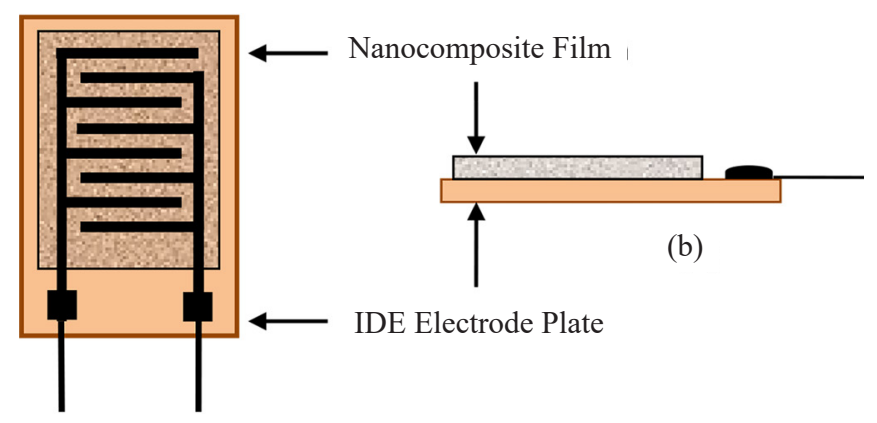

(a)

Figure 1: Schematic representation of IDE sensor, with (a) top view, and (b) side view.

\subsection{Ethanol Sensing}

The gas sensing properties of PPy/MWCNT composite at room temperature were tested in indigenously designed gas sensing equipment consisting of $500 \mathrm{ml}$ glass container with gas inlet and outlet port. The DC resistance was recorded by Keithley 2000 digital multimeter.

The response magnitude $\mathrm{S}$ of sensor was defined as:

$$
S(\%)=\left(\frac{\mathrm{R}_{\mathrm{g}}-\mathrm{R}_{\mathrm{a}}}{\mathrm{R}_{\mathrm{a}}}\right) * 100
$$


where $R_{a}$ and $R_{g}$ represented resistance in air and that of gas for different concentrations, respectively.

\section{RESULTS AND DISCUSSION}

\subsection{Characterisations of Samples}

The FESEM images of pure PPy, PPy/MWCNT nanocomposite and functionalised MWCNT are shown in Figure 2. The FESEM image of MWCNTs shown in Figure 2(a) demonstrates several nanotubes with diameter $\sim 10 \mathrm{~nm}$, which are loosely entangled. The FESEM image of PPy as observed in Figure 2(b) has cauliflower-like morphology with grain size $100-400 \mathrm{~nm} .{ }^{23}$ The FESEM images of Figure 2(c) and Figure 2(d) for PPy/MWCNT composites shows the same morphology as for pristine PPy with reduced grain size. The grain size observed for PPy/MWCNT5 sample has in the range 150-200 nm, while for PPy/MWCNT50 sample, it is $80-100 \mathrm{~nm}$. This may due to the increase in MWCNT content. The MWCNTs in the PPy/MWCNT composites are not visible due to excess aggregation of PPy on MWCNTs due to more composition of PPy as compared to MWCNTs.
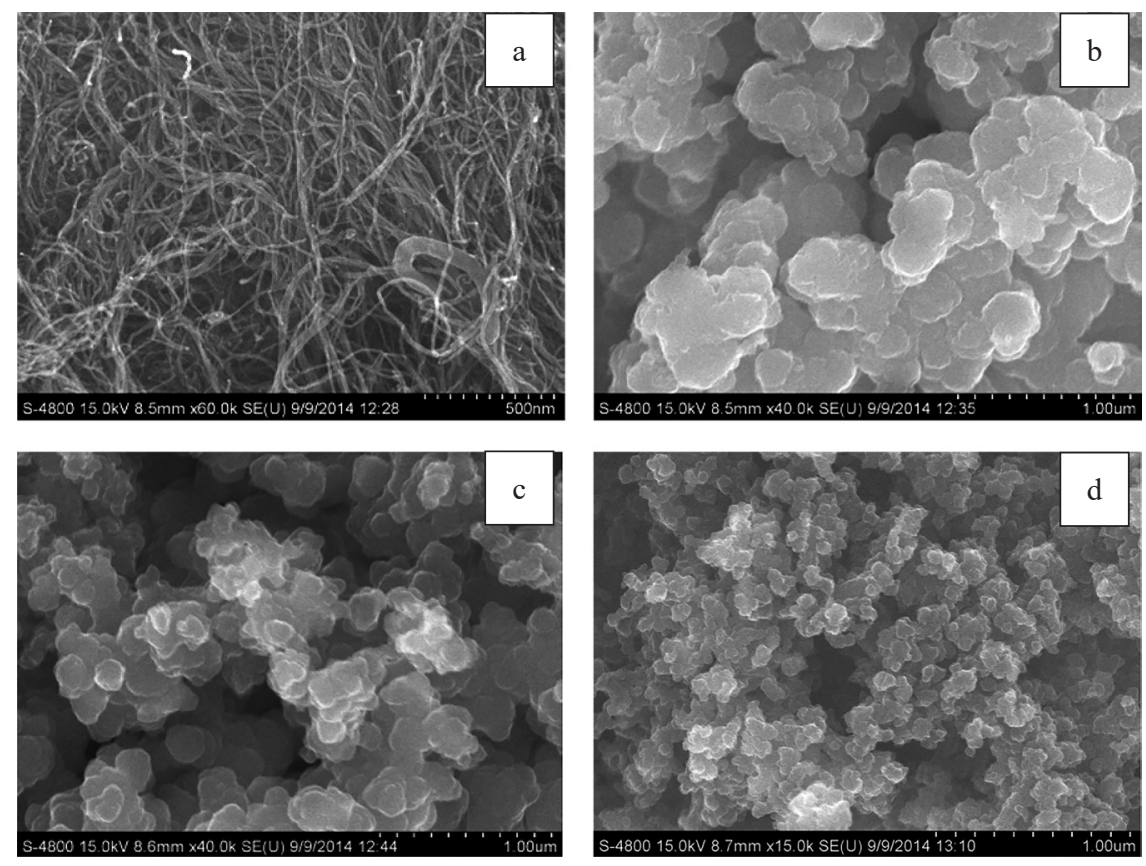

Figure 2: FESEM images of (a) MWCNT, (b) PPy, (c) PPy/MWCNT5 and (d) PPy/ MWCNT50. 
The XRD pattern of PPy, PPy/MWCNT nanocomposites and MWCNTs are shown in Figure 3. The pristine PPy exhibited a broad diffraction peak at $2 \theta=15^{\circ} \mathrm{C}-33^{\circ} \mathrm{C}$, which showed that the PPy is in amorphous nature. The spectra for MWCNT showed the peaks at $2 \theta=24.4^{\circ}$ and $43^{\circ}$ corresponds to (002) and (100) reflection of carbon atoms. ${ }^{23}$ The XRD pattern of PPy/MWCNT showed the characteristics of PPy as well as MWCNT. The overlapped diffraction peaks of MWCNT with PPy depict the formation of PPy/MWCNT nanocomposite by insitu polymerisation synthesis process. ${ }^{24}$

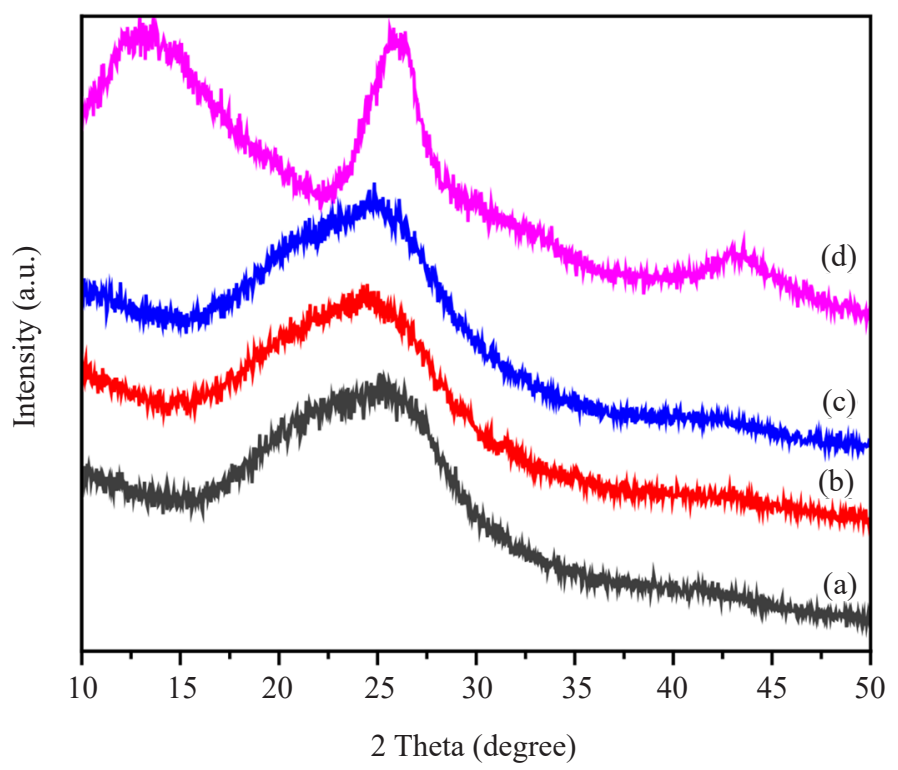

Figure 3: XRD patterns of (a) PPy, (b) PPy/MWCNT5, (c) PPy/MWCNT10 and (d) MWCNT.

The FTIR spectra for pristine PPy, MWCNT and PPy/MWCNT nanocomposites are shown in Figure 4. The FTIR spectra of MWCNT exhibited two intense peaks at $1390 \mathrm{~cm}^{-1}$ and $1535 \mathrm{~cm}^{-1}$ which attributed to bend vibration of the $\mathrm{O}-\mathrm{H}$ group and carbonyl, respectively. The small intense peak at $\sim 1700 \mathrm{~cm}^{-1}$ corresponded to the $\mathrm{C}=\mathrm{O}$ stretching vibration mode, representing the formation of the carboxylic groups. A broad peak at $3460 \mathrm{~cm}^{-1}$ represents a characteristic of an O-H stretch, which is observed due to alcoholic or phenolic carboxylic groups. This result showed that the MWCNTs successfully oxidised into carboxylated CNTs. ${ }^{25}$ In the spectra of pristine PPy, the peaks obtained at $1562 \mathrm{~cm}^{-1}$ and $1395 \mathrm{~cm}^{-1}$ corresponded to the anti-symmetric and symmetric C-C stretching vibration respectively in pyrrole ring. The small peak at $1300 \mathrm{~cm}^{-1}$ is related to the $\mathrm{C}-\mathrm{N}$ in-plane, and the peaks at $1210 \mathrm{~cm}^{-1}$ and $1055 \mathrm{~cm}^{-1}$ are associated to the 
$\mathrm{C}-\mathrm{H}$ bending modes while the band for $\mathrm{C}-\mathrm{H}$ out-of-plane deformation vibration was observed at $898 \mathrm{~cm}^{-1} .{ }^{24}$ The peak appearing at $680 \mathrm{~cm}^{-1}$ corresponds to ring deformation. ${ }^{26}$ It is observed that all the peaks appearing in the fingerprint region of PPy are also observed in the FTIR spectra of PPy/MWCNT nanocomposites. This indicates that the main constituents of PPy and its nanocomposite with MWCNTs have the same chemical structure.

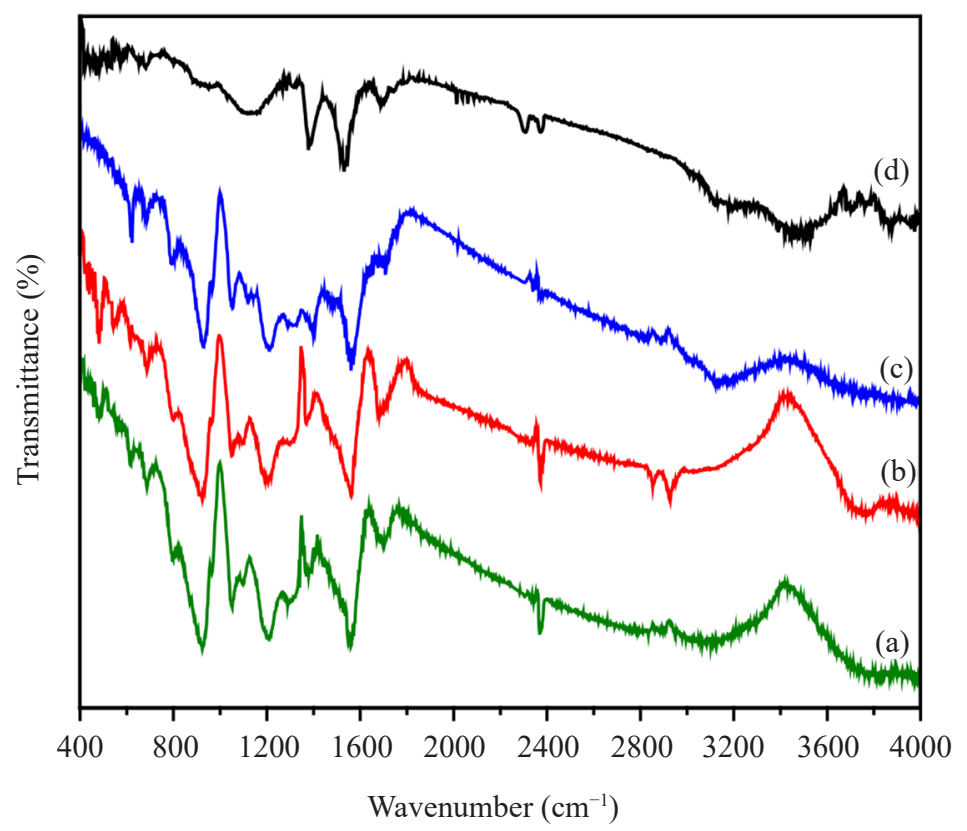

Figure 4: FTIR spectrum of (a) PPy, (b) PPy/MWCNT5, (c) PPy/MWCNT50 and (d) MWCNT.

TGA measurements were carried out to investigate the effect of incorporation of functionalised MWCNT on thermal stability of PPy/MWCNT. The results for few PPy/MWCNT nanocomposites are shown in Figure 5. For comparison, the TGA analysis of pure PPy and functionalised MWCNT are also shown in this figure. It is observed that the pure PPy was stable in the temperature range up to $250^{\circ} \mathrm{C}$ and shows only about $12 \%$ mass loss. This loss in weight may occur due to dehydration of the PPy. However, a rapid weight loss takes place after $250^{\circ} \mathrm{C}$, which corresponded to the complete degradation and decomposition of PPy at different polymerisation stage. ${ }^{24}$ The functionalised MWCNTs are comparatively more stable and showing no dramatic decomposition, with a $18 \%$ mass loss occurred in observed temperature range. ${ }^{27}$ This loss may due to the decomposition of functional groups (such as $\mathrm{C}=\mathrm{C}$ and $\mathrm{COOH}$ ) present in MWCNTs. As compared to pure $\mathrm{PPy}$, the $\mathrm{PPy} / \mathrm{MWCNT}$ nanocomposite samples showed slower 
decomposition. The thermal stability of PPy/MWCNT samples increased with the increase of the MWCNTs content in PPy. This indicated that the addition of the functionalised CNT had improved the thermal stability of PPy/MWCNT nanocomposites and there exist interfacial interaction between MWCNTs with PPy shell. ${ }^{25,27}$ It is important to point out that MWCNT decomposed in one stage whereas PPy decomposed in two stages until it was completely decomposed at approximately $660^{\circ} \mathrm{C} .^{28}$

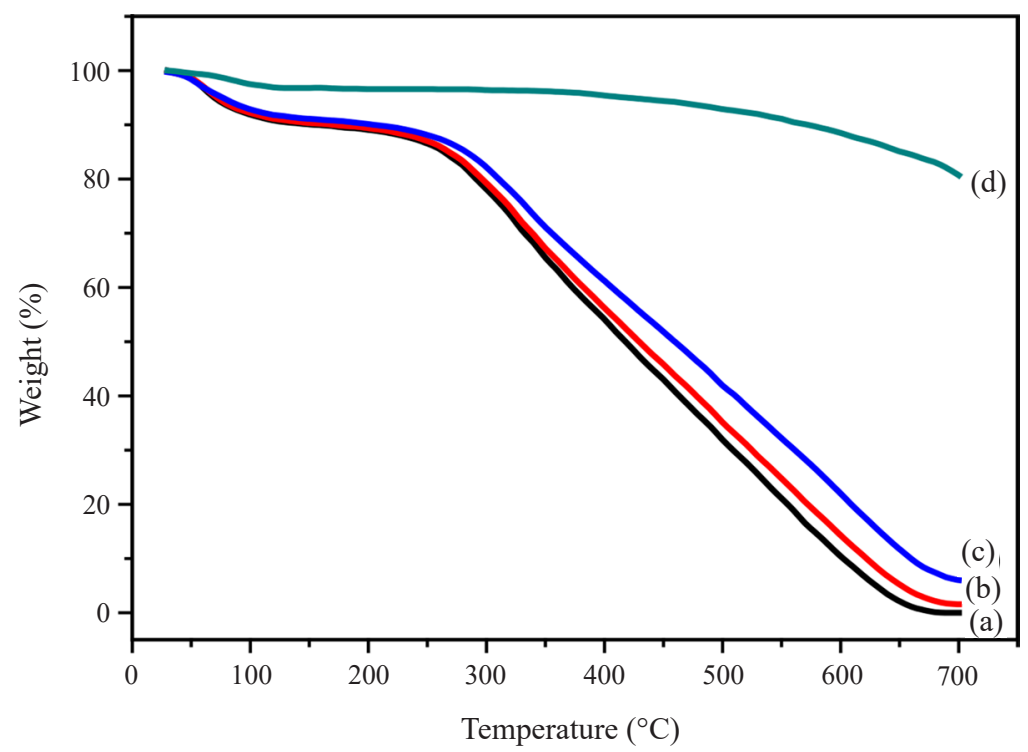

Figure 5: TGA curves of (a) pure PPy, (b) PPy/MWCNT5, (c) PPy/MWCNT25 and (d) MWCNT.

\subsection{Ethanol Sensing by Resistive Response}

At the beginning of test, the sensor was exposed to air in glass container and the measured resistance of the sensor was equal to $R_{a}$. The sensor was then exposed to ethanol vapour by injecting desired ethanol vapour in glass container with syringe pump. The change in resistance was recorded as $\mathrm{R}_{\mathrm{g}}$. It was observed that the resistance of the sensor changed abruptly on exposure to ethanol and returns to some steady value. Thus, a sharp spike was observed on exposure of sensor with gas. The ethanol vapour was then withdrawn from glass container using vacuum pump, so as to expose the sensor to air again. The measured resistance was returned to almost its original value, $R_{a}$.

All the samples were exposed to $0.02 \%, 0.1 \%, 0.2 \%, 0.3 \%, 0.4 \%, 0.6 \%, 0.8 \%$ and $1.0 \%$ volume of ethanol vapour concentrations. The response of the sensors for 
all nanocomposite samples was calculated in terms of percentage sensitivity using Equation 1 . The sensitivity values were averaged over three cycles of measurement and are listed in Table 1. The variation of sensitivity with variation in ethanol concentration is shown in Figure 6.

Table 1: Sensitivity of PPy and PPy/MWCNT nanocomposites to different ethanol vapour concentrations.

\begin{tabular}{cccccc}
\hline \multirow{2}{*}{$\begin{array}{c}\text { \% Volume } \\
\text { of ethanol }\end{array}$} & \multicolumn{5}{c}{ \% Sensitivity } \\
\cline { 2 - 6 } & PPy & PPy/MWCNT 5 & PPy/MWCNT 10 & PPy/MWCNT 25 & PPy/MWCNT 50 \\
\hline 0.02 & 1.47 & 2.10 & 3.07 & 5.29 & 4.87 \\
0.1 & 2.53 & 5.39 & 7.94 & 13.40 & 11.64 \\
0.2 & 3.86 & 7.16 & 9.35 & 15.93 & 14.70 \\
0.3 & 4.72 & 8.01 & 10.50 & 17.00 & 15.92 \\
0.4 & 4.46 & 7.77 & 11.40 & 18.54 & 17.31 \\
0.6 & 5.19 & 8.47 & 12.01 & 21.72 & 19.29 \\
0.8 & 5.72 & 8.60 & 14.05 & 22.11 & 20.22 \\
1.0 & 6.65 & 9.65 & 14.20 & 24.01 & 22.08 \\
\hline
\end{tabular}

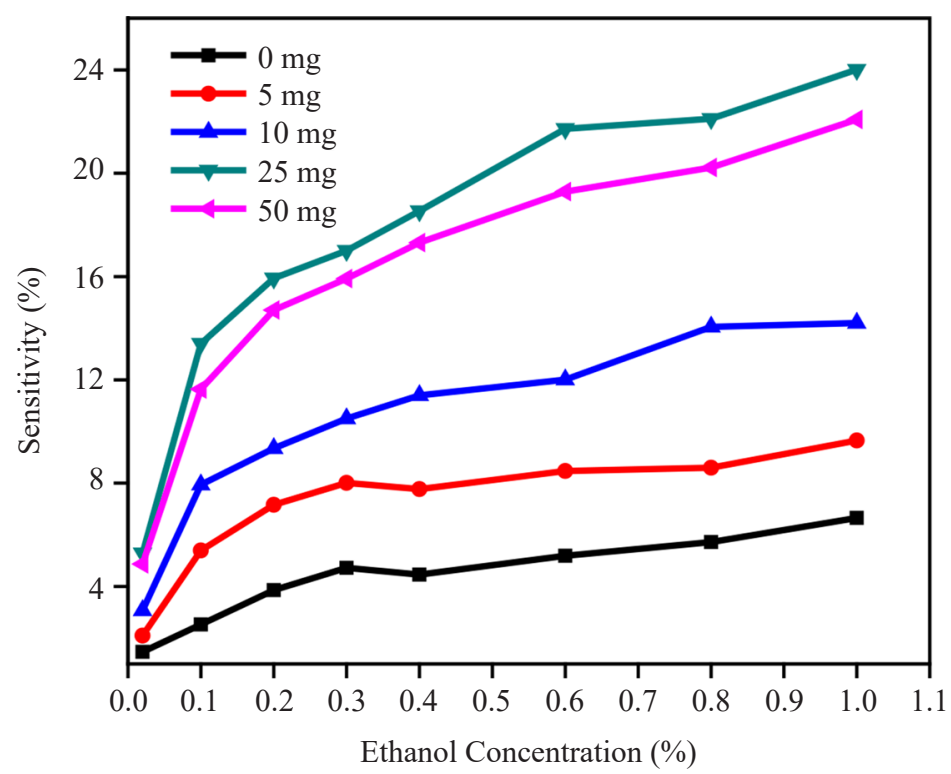

Figure 6: The sensor response as a function of ethanol concentration for PPy and PPy/ MWCNT samples at various MWCNT contents. 
The sensitivity increases rapidly with increase in values up to $0.3 \%$ of ethanol concentration and increases slowly thereafter. It was observed from Figure 7 that the sensitivity varies with MWCNT content indicating that sensitivity of the composite sensors influenced by varying the MWCNT content in the PPy. It is predicted from the Figure 7 that response is maximum in the range $25-40 \mathrm{mg}$ MWCNT content. The trend of change in sensitivity with MWCNT content was similar for all concentrations of ethanol vapour.

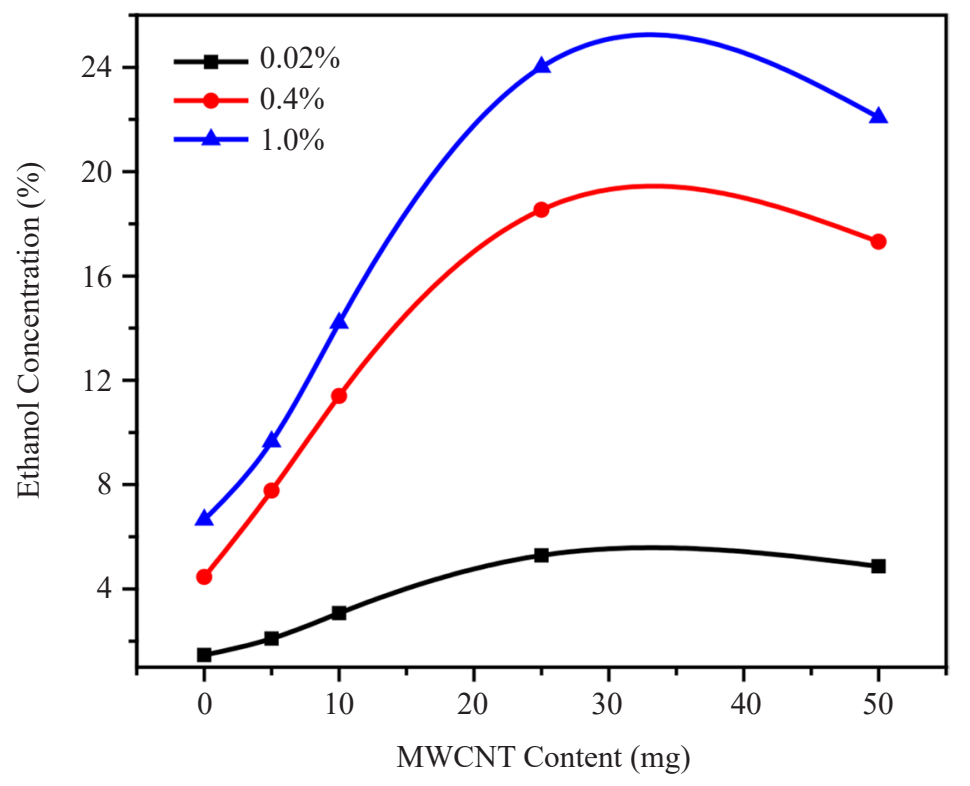

Figure 7: The sensor response as a function of the MWCNT content for different ethanol concentrations.

It is worth to note that all the sensors exhibit a strong positive vapour coefficient (PVC) effect when exposed to ethanol vapours. This means that the resistance of the sensors increases with ethanol vapour sorption and decreases with its desorption in air. The response for typical sensor is shown in Figure 8. It can be observed that the response time is about $5 \mathrm{~s}$ while the recovery value is about $15 \mathrm{~s}$. Such kind of transient response can easily operate electronic switching devices and can be used to develop VOC electronic sensing unit. The values reported in the Table 1 reveal that the sensitivity for pure PPy sample is less compared with all other nanocomposites and maximum for the nanocomposite having $25 \mathrm{mg}$ MWCNT content. The sensitivity decreases for nanocomposites having higher MWCNT content. 


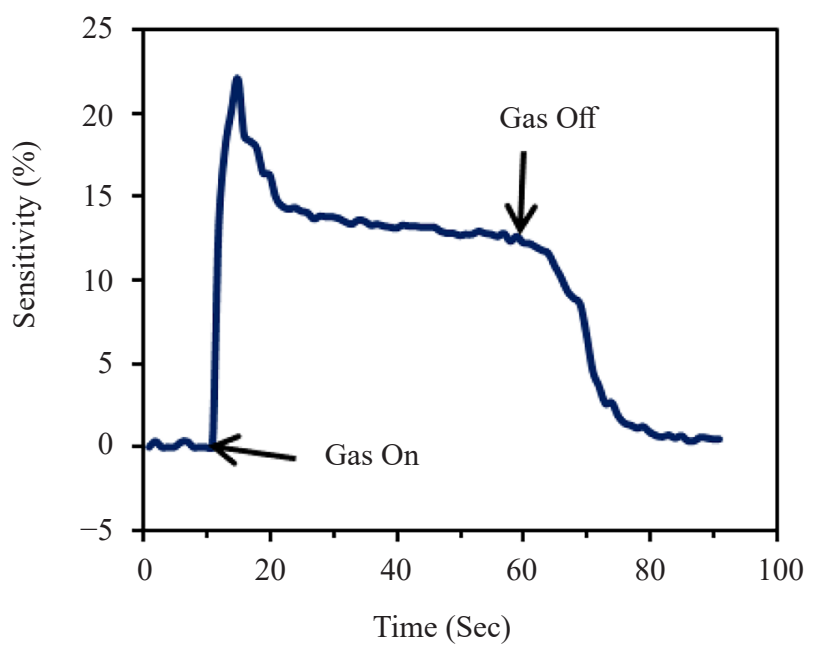

Figure 8: Ethanol response (\%) vs. time curve of PPy/MWCNT25 sensor at room temperature.

The thickness of the polymer layer deposited on the MWCNT has a significant effect on the sensitivity of composite sensors. The sensing mechanism of pristine MWCNT based sensor depends upon electron transfer mechanism that occurs due to simple adsorption of VOC molecules on MWCNT surface. ${ }^{29}$ These VOC molecules on MWCNT surface form a thin insulating coating, which is, in turn, forcing the electronic transfer by tunnelling at MWCNT-MWCNT junction. ${ }^{30}$

The sensing mechanism of polymer coated MWCNT nanocomposites is based on the swelling of this thin polymer layer on MWCNT due to solvent molecules diffusion, which is assumed to be responsible for the strong PVC response observed. An increase in MWCNT-MWCNT junction gap produced due to swelling of VOC molecules by polymer can cause an increase in resistance as represented in Figure 9. In the presence of gap at MWCNT-MWCNT produced due to swelling of VOC molecules, the electron can jump from MWCNT to MWCNT provided that they have enough potential energy to do so and if the gap is less than few tens of nm. ${ }^{31}$ Thus, the electron transportation is mainly produced by quantum tunnelling, which in turn leads the contact resistance between MWCNT to increase exponentially with the gap at MWCNT-MWCNT junctions according to the quantum resistive conduction model of Equation 2:

$$
\frac{\Delta R}{R_{0}}=a e^{b \Delta Z}
$$

where $\Delta R / R_{0}$ is the tunnel relative resistance variation, whereas $a$ and $b$ are positive constants and $\Delta Z$ is the gap variation between MWCNTs. ${ }^{32}$ 


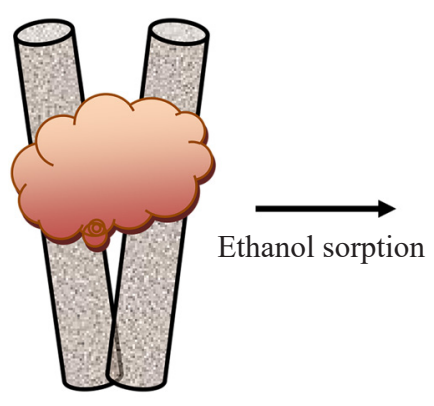

(a)

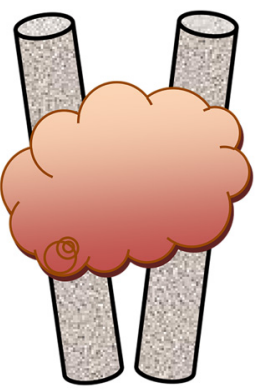

(b)

Figure 9: Schematic representation of sensing mechanism due to junction gap variation (a) in dry air, (b) in presence of ethanol vapour.

Therefore, the swelling of PPy layer caused by ethanol molecules affects the conduction by tunnelling as the conductive network formed by MWCNTs is gradually disconnected. However, increase in PPy layer thickness can increase diffusion time of ethanol molecules at MWCNT-MWCNT junction, consequently slowing down their disconnecting dynamics. ${ }^{33}$ The decrease in thickness of polymer over MWCNT with increase of MWCNT content leads to decrease in diffusion time of VOC molecules; however the probability of disconnection of CNTs is less. Therefore, the sensor with polymer nanocomposite having less MWCNT content has higher sensitivity than nanocomposite has higher MWCNT content. ${ }^{29}$ It is observed that for the PPy/MWCNT25 nanocomposites under study, the sensor corresponding to $25 \mathrm{mg}$ MWCNT content has highest sensitivity due to best compromise between junction gap and diffusion time.

\section{CONCLUSION}

APPy/MWCNTnanocomposite for differentMWCNT content has been successfully synthesised by in-situ chemical oxidative polymerisation. FESEM images conform that PPy has successfully formed on the surface of MWCNTs. XRD and FTIR spectrum revealed the incorporation of MWCNT into the PPy matrix. TGA analysis showed that the thermal stability of polypyrrole improved significantly by the formation of composite with MWCNT. The PPy/MWCNT nanocomposite sensors show excellent sensitivity to ethanol vapours at room temperature with fast response-recovery. The content of MWCNT in the PPy significantly affects the sensitivity towards ethanol. The most sensitive PPy/MWCNT nanocomposites sensor to ethanol vapours obtained with $25 \mathrm{mg}$ MWCNT content and found to be the best compromise between junction gap and diffusion time. 


\section{ACKNOWLEDGEMENTS}

The authors are grateful to North Maharashtra University, Jalgaon, India for financial support through the Vice-Chancellor Research Motivation Scheme (VCRMS) project scheme (NMU/11A/VCRMS/Budget-2013-14/Science/167/2014). The authors are also thankful to University Grants Commission-Department of Atomic Energy (UGC-DAE) Consortium for Scientific Research Centre, Indore for providing the characterisation facilities.

\section{REFERENCES}

1. Hsiao, A.-E. et al. (2012). Decoration of multi-walled carbon nanotubes by polymer wrapping and its application in MWCNT/polyethylene composites. Nanosci. Res. Lett., 7, 240, https://doi.org/10.1186/1556-276X-7-240.

2. Mittal, M. \& Kumar, A. (2014). Carbon nanotube (CNT) gas sensors for emissions from fossil fuel burning. Sens. Actuat. B Chem., 203, 349-362, https://doi.org/10.1016/j.snb.2014.05.080.

3. Li, L. et al. (2012). Fabrication and characterization of free-standing polypyrrole/graphene oxide nanocomposite paper. J. Nanopart. Res., 14, 908, https://doi.org/10.1007/s11051-012-0908-3.

4. Whitby, R. L. et al. (2011). Morphological effects of single-layer graphene oxide in the formation of covalently bonded polypyrrole composites using intermediate diisocyanate chemistry. J. Nanopart. Res., 13, 4829-4837, https://doi.org/10.1007/s11051-011-0459-z.

5. Sirivisoot, S., Pareta, R. \& Webster, T. J. (2011). Electrically controlled drug release from nanostructured polypyrrole coated on titanium. Nanotechnol., 22, 085101, https://doi.org/10.1088/0957-4484/22/8/085101.

6. Yalçınkaya, S. et al. (2010). Electrochemical synthesis and characterization of polypyrrole/chitosan composite on platinum electrode: Its electrochemical and thermal behaviors. Carbohydr. Polym., 79, 908-913, https://doi. org/10.1016/j.carbpol.2009.10.022.

7. Balint, R., Cassidy, N. J. \& Cartmell, S. H. (2014). Conductive polymers: Towards a smart biomaterial for tissue engineering. Acta Biomater, 10, 2341-2353, https://doi.org/10.1016/j.actbio.2014.02.015.

8. Baibarac, M., Baltog, I. \& Lefrant, S. (2011). Recent progress in synthesis, vibrational characterization and applications trend of conjugated polymers/ carbon nanotubes composites. Curr. Org. Chem., 15, 1160-1196, https://doi. org/10.2174/138527211795203022. 
9. Lee, Y.-K. et al. (2010). Polypyrrole-carbon nanotube composite films synthesized through gas-phase polymerization. Synth. Met., 160, 814-818, https://doi.org/10.1016/j.synthmet.2010.01.028.

10. Chen, H. et al. (2011). Multilayered polypyrrole-coated carbon nanotubes to improve functional stability and electrical properties of neural electrodes. $J$. Phys. Chem. C, 115, 5492-5499, https://doi.org/10.1021/jp111498e.

11. Han, Y. et al. (2012). Ternary phase interfacial polymerization of polypyrrole/ MWCNT nanocomposites with core-shell structure. Synth. Met., 162, $753-$ 758, https://doi.org/10.1016/j.synthmet.2012.03.001.

12. Rana, S. \& Cho, J. W. (2011). Core-shell morphology and characterization of carbon nanotube nanowires click coupled with polypyrrole. Nanotechnol., 22, 275609, https://doi.org/10.1088/0957-4484/22/27/275609.

13. Park, S. J., Kwon, O. S. \& Jang, J. (2013). A high-performance hydrogen gas sensor using ultrathin polypyrrole-coated CNT nanohybrids. Chem. Comm., 49, 4673-4675, https://doi.org/10.1039/C3CC41020J.

14. Wu, T.-M., Chang, H.-L. \& Lin, Y.-W. (2009). Synthesis and characterization of conductive polypyrrole/multi-walled carbon nanotubes composites with improved solubility and conductivity. Compos. Sci. Technol., 69, 639-644, https://doi.org/10.1016/j.compscitech.2008.12.010.

15. Dragonieri, S. et al. (2012). An electronic nose distinguishes exhaled breath of patients with Malignant Pleural Mesothelioma from controls. Lung Canc., 75, 326-331, https://doi.org/10.1016/j.lungcan.2011.08.009.

16. Peng, G. et al. (2010). Detection of lung, breast, colorectal, and prostate cancers from exhaled breath using a single array of nanosensors. Br. J. Can., 103, 542, https://doi.org/10.1038/sj.bjc.6605810.

17. Arasaradnam, R. P. et al. (2011). Insights into 'fermentonomics': Evaluation of volatile organic compounds (VOCs) in human disease using an electronic 'e-nose.' J. Med. Eng. Technol., 35, 87-91, https://doi.org/10.3109/030919 02.2010 .539770 .

18. Goldoni, A. et al. (2009). Sensing gases with carbon nanotubes: A review of the actual situation. J. Phys. Condens. Matt., 22, 013001, https://doi. org/10.1088/0953-8984/22/1/013001.

19. Tisch, U. \& Haick, H. (2010). Nanomaterials for cross-reactive sensor arrays. MRS Bull., 35, 797-803, https://doi.org/10.1557/mrs2010.509.

20. Shin, J. et al. (2013). Thin-wall assembled $\mathrm{SnO} 2$ fibers functionalized by catalytic Pt nanoparticles and their superior exhaled-breath-sensing properties for the diagnosis of diabetes. Adv. Funct. Mater., 23, 2357-2367, https://doi.org/10.1002/adfm.201202729.

21. Lu, J. et al. (2010). Polyaniline nanoparticle-carbon nanotube hybrid network vapour sensors with switchable chemo-electrical polarity. Nanotechnol., 21, 255501, https://doi.org/10.1088/0957-4484/21/25/255501. 
22. Benlikaya, R. et al. (2014). The enhanced alcohol sensing response of multiwalled carbon nanotube networks induced by alkyl diamine treatment. Sens. Actuat. B Chem., 201, 122-130, https://doi.org/10.1016/j. snb.2014.04.057.

23. Sahoo, N. G. et al. (2007). Polypyrrole coated carbon nanotubes: Synthesis, characterization, and enhanced electrical properties. Synth. Met., 157, 374 379, https://doi.org/10.1016/j.synthmet.2007.04.006.

24. Manivel, P. et al. (2014). Rheological behavior and electrical properties of polypyrrole/thermally reduced graphene oxide nanocomposite. Coll. Surf. A Physicochem. Eng. Asp., 441, 614-622, https://doi.org/10.1016/j. colsurfa.2013.10.031.

25. Liu, P., Wang, X. \& Li, H. (2013). Preparation of carboxylated carbon nanotubes/polypyrrole composite hollow microspheres via chemical oxidative interfacial polymerization and their electrochemical performance. Synth. Met., 181, 72-78, https://doi.org/10.1016/j.synthmet.2013.08.010.

26. Mahore, R. P., Burghate, D. K. \& Kondawar, S. B. (2014). Development of nanocomposites based on polypyrrole and carbon nanotubes for supercapacitors. Adv. Mater. Lett., 5, 400-405, https://doi.org/10.5185/ amlett.2014.amwc.1038.

27. Zhang, B. et al. (2011). A facile synthesis of polypyrrole/carbon nanotube composites with ultrathin, uniform and thickness-tunable polypyrrole shells. Nanosc. Res. Lett., 6, 431, https://doi.org/10.1186/1556-276X-6-431.

28. Hermas, A. et al. (2012). In situ electropolymerization of conducting polypyrrole/carbon nanotubes composites on stainless steel: Role of carbon nanotubes types. Progr. Org. Coat., 75, 404-410, https://doi.org/10.1016/j. porgcoat.2012.07.006.

29. Kumar, B., Castro, M. \& Feller, J.-F. (2013). Quantum resistive vapour sensors made of polymer coated carbon nanotubes random networks for biomarkers detection. Chem. Sens., 3, 1-7.

30. Lu, J. et al. (2011). Chemo-sensitivity of latex-based films containing segregated networks of carbon nanotubes. Sens. Actuat. B Chem., 155, $28-$ 36, https://doi.org/10.1016/j.snb.2010.11.017.

31. Zamborini, F. P. et al. (2002). Electron hopping conductivity and vapor sensing properties of flexible network polymer films of metal nanoparticles. J. Am. Chem. Soc., 124, 8958-8964, https://doi.org/10.1021/ja025965s.

32. Feller, J.-F. et al. (2011). Novel architecture of carbon nanotube decorated poly (methyl methacrylate) microbead vapour sensors assembled by spray layer by layer. J. Mater. Chem., 21, 4142-4149, https://doi.org/10.1039/ C0JM03779F. 
33. Lu, J. et al. (2009). Vapour sensing with conductive polymer nanocomposites (CPC): Polycarbonate-carbon nanotubes transducers with hierarchical structure processed by spray layer by layer. Sens. Actuat. B Chem., 140, 451-460, https://doi.org/10.1016/j.snb.2009.05.006. 